\title{
Pharmacodynamics and Cellular Uptake of Peimine and Peiminine in Inflammatory Model Non-Small-Cell Lung Cancer Epithelial Cells (A549)
}

\author{
Zhan-Ke Chen $\mathbb{D}^{1,2,3}$ Di Zhao, ${ }^{3,4,5}$ Su-Xiang Feng $\mathbb{D}^{3,4,5}$ and Jiangyan Xu $\mathbb{D}^{3}$ \\ ${ }^{1}$ Shandong University of Traditional Chinese Medicine, Jinan, China \\ ${ }^{2}$ The First Affiliated Hospital of Henan University of Chinese Medicine, Zhengzhou 450000, China \\ ${ }^{3}$ Henan University of Chinese Medicine, Zhengzhou 450046, China \\ ${ }^{4}$ Henan Key Laboratory of Chinese Medicine for Respiratory Disease, Henan University of Chinese Medicine, Zhengzhou, \\ Henan 450046, China \\ ${ }^{5}$ Co-Construction Collaborative Innovation Center for Chinese Medicine and Respiratory Diseases By \\ Henan \& Education Ministry of P.R. Zhengzhou, Zhengzhou 450046, China
}

Correspondence should be addressed to Su-Xiang Feng; fengsx221@163.com and Jiangyan Xu; xujiangyan2008@163.com

Received 17 August 2021; Accepted 15 December 2021; Published 7 February 2022

Academic Editor: Samuel Martins Silvestre

Copyright $\odot 2022$ Zhan-Ke Chen et al. This is an open access article distributed under the Creative Commons Attribution License, which permits unrestricted use, distribution, and reproduction in any medium, provided the original work is properly cited.

Peimine and peiminine are isosteroidal alkaloids with multiple biological activities, such as anticancer and anti-inflammatory activities, but their cellular uptake and pharmacodynamics are unclear. In this study, a rapid and sensitive ultra-performance liquid chromatography-tandem mass spectrometry (UPLC-MS/MS) method was developed for the simultaneous quantification of peimine and peiminine concentrations in A549 cells. In the pharmacodynamic study, the selected inflammatory cytokines were IL-8, MMP-9, and TIMP-1. The results demonstrated that all calibration curves exhibited good linearity $(r>0.9970)$. The RSDs of intraday and interday precision and accuracy were less than $6.73 \%$ and $1.76 \%$ and $7.73 \%$ and $3.05 \%$ for peimine and peiminine, respectively. Moreover, the average analytic recoveries ranged from $83.85 \%$ to $113.67 \%$, and the matrix effect was within $95.05 \%-$ $111.29 \%$. The uptake experiment showed a time-dependent characteristic in the A549 cells. The combination group had increased uptake and had a longer $T_{\max }$ than the single group. In the experimental pharmacodynamics groups, the anti-inflammatory effects of the $100.0 \mu \mathrm{g} / \mathrm{mL}$ combination group were the most obvious. This investigation, for the first time, explores the cellular uptake profiles and pharmacodynamics of peimine and peiminine in A549 cell lines.

\section{Introduction}

Targeted drug therapies are receiving more and more attention. Cellular uptake plays an indispensable role in the research of drug disposal in cells and in predicting and evaluating drug efficacies. Peimine and peiminine are isosteroidal alkaloids and are the main biologically active components of Fritillariae Thunbergii Bulbus (FTB) [1]. FTB is a significant Chinese medicine that has been clinical for thousands of years, with bitter and cold properties that clear heat, resolve phlegm, relieve cough, detoxify, and eliminate carbuncles [2,3]. Modern pharmacological studies show that peimine and peiminine display analgesic [4], anti- inflammatory [5], and antitumor [6] biological activities. They also relax smooth muscles [7]. Peimine significantly inhibits the secretion of proinflammatory cytokines (e.g., TNF- $\alpha$, IL-6, and IL-1 $\beta$ ), increases anti-inflammatory cytokines (e.g., IL-10), and inhibits the production of lipopolysaccharide (LPS) induced inflammatory cytokines. It blocks the signaling pathways of the extracellular signalregulating kinase (MAPK) and nuclear factor-kappa B (NF$\mathrm{kB})[8,9]$. Peiminine reportedly has a strong anti-inflammatory effect on a variety of diseases, making it a potential therapeutic drug for pulmonary diseases [10], osteoarthritis (OA) [11], Parkinson's disease (PD) [12], and mast cellassociated allergic inflammatory diseases [13]. In mouse OA 
chondrocytes, peiminine reduces IL- $1 \beta$ by inhibiting AKT/ $\mathrm{NF}-\mathrm{kB}$ and activating Nrf2/HO-1 to induce the inflammatory response [11]. Also, peiminine inhibited neuroinflammation to protect dopaminergic neurons in the LPSinduced PD rat model and significantly reduced the production of proinflammatory mediators in BV-2 cells [12].

Cellular pharmacokinetics is an emerging branch of classical pharmacokinetics that has received extensive attention for its role in drug evaluation and development in recent years [14]. A growing number of reports indicate that pharmacokinetic research based on plasma drug concentrations cannot fully elucidate the pharmacological effects of drugs in some tissues (e.g., tumors or brain tissues). Furthermore, it is difficult to truly and effectively predict drug efficacy in vivo [15]. The drug concentration around the intracellular target can provide a truer reflection of drug efficacy since the correlation between the drug concentration in the cell and the toxic reaction is stronger than in the plasma [16-18]. This is a microscopic perspective of pharmacokinetics that considers the cell as a whole to quantitatively analyze the dynamics of drug uptake, distribution, metabolism, and excretion within the cell to assess the efficacy of the drug in the target cell [19]. At present, there have been studies on the pharmacokinetics of peimine and peiminine in rats and beagles $[20,21]$. Cellular uptake is one of the central challenges in chemical biology and beyond. With the objective to find conceptually innovative ways to enter cells, cyclic oligochalcogenides (COCs) are emerging as powerful tools [22]. Cellular uptake uses live cells to examine the effects of compounds on receptor or transporter systems. It can determine a compound's potency and efficacy. It can also be used to measure a compounds transport into cells or efflux from cells. Cell experiments in vitro have shown that peimine displays anti-inflammatory and analgesic properties at the cellular level [5]. No method has been reported for the simultaneous determination of peimine and peiminine in cell samples, nor for its application in cellular uptake studies, and only a few studies have been combined with pharmacodynamics.

A549 cells, characterized by alveolar type II epithelial cells, are the preferred cells for establishing in vitro cell models of acute lung injury [23]. TNF- $\alpha$ stimulates A549 cells to induce an inflammatory response, which may be related to the activation of excessive protease, the increase of interleukin levels, and the induction of airway epithelial cells into goblet cells, increasing inflammatory gene expression [24]. Thus, we used pharmacodynamics to study the effects of peimine and peiminine o TNF- $\alpha$ induced A549 cell inflammation models using the inflammatory cytokines IL8, MMP-9, and TIMP-1. In addition, we developed and validated a specific and sensitive UPLC-MS/MS method for the simultaneous determination of peimine and peiminine concentrations in A549 cell lysate and successfully applied this method to the study of cellular pharmacokinetic characteristics. This paper provides a useful reference of the pharmacologic mechanisms and dynamic laws in cells and a theoretical basis for the safe and effective use of peimine and peiminine.

\section{Materials and Methods}

2.1. Material and Reagents. Peimine and peiminine were purchased from the National Institutes for Food and Drug Control (Beijing, China). Carbamazepine (internal standard; IS) was obtained from Shanghai Yuan Ye Bio-Technology Co., Ltd. (Shanghai, China). These reference substances had a purity of $>98 \%$. The raw material peimine (purity $\geq 90 \%$ ) was obtained from Xi'an Hui Lin Bio-Technology Co., Ltd. (Shaanxi, China). The raw material peiminine (purity $\geq 90 \%$ ) was provided by Chengdu Master Biotechnology Co., Ltd. (Chengdu, China). Methanol and acetonitrile were provided by the Tedia Company (USA). Ammonium formate was obtained from Fisher (USA). All solutions were mass spectrum grade. Fetal bovine serum, trypsin, methyl thiazole tetrazolium (MTT), and Roswell Park Memorial Institute (RPMI) medium 1640 were purchased from Beijing Solarbio Science and Technology Co., Ltd. (Beijing, China). TNF- $\alpha$ was purchased from Peprotech (USA). The ultrapure water was produced by the Millipore Mill-Q system (Bedford, MA, USA). Human IL-8, MMP-9, and TIMP-1 ELISA kits were purchased from Baxter Biological Co., Ltd. The A549 cell line was obtained from Shanghai Institutes for Biological Sciences (Shanghai, China).

2.2. Cell Culture. A549 cell lines were cultured in RPMI 1640 medium that was supplemented with $10 \%$ fetal bovine serum (FBS) and then incubated at $37^{\circ} \mathrm{C}$ under a humidified incubator of $5 \% \mathrm{CO}_{2}$. The cell culture medium was changed every 2 days and passaged every 3 days at a ratio of $1: 3$. The A549 cells grew in a logarithmic period and were mixed into $2 \times 10^{6} / \mathrm{ml}$ in the cell culture medium. After the cells were mixed with cryopreservation solution (DMSO : FBS: RPMI Medium $1640(1: 2: 7))$, they were kept at $4^{\circ} \mathrm{C}$ for $2 \mathrm{~h}$, followed by $-20^{\circ} \mathrm{C}$ for $6 \mathrm{~h}$, and then stored in liquid nitrogen.

2.3. MTT Assay for Cell Viability. A549 cells were seeded at a density of $2 \times 10^{4} / \mathrm{mL}$ in a 96-well ordinary flat-bottom plate at $100 \mu \mathrm{L}$ per well. Once the cells reached $80 \%$, they were grouped as follows: blank group (K, 10\% FBS complete medium), model group ( $\mathrm{M}$, containing $10 \mathrm{ng} / \mathrm{mL} \mathrm{TNF}-\alpha$, $10 \%$ FBS complete medium), 12 normal cell experimental groups (4 different peimine concentrations, 4 different peiminine concentrations, and 4 different peimine + peiminine concentrations), and 12 model cell experimental groups (4 different peimine concentrations, 4 different peiminine concentrations, and 4 different peimine + peiminine concentrations). The concentrations of peimine and peiminine in each of the 4 groups were $25 \mu \mathrm{g} / \mathrm{mL}$, $50 \mu \mathrm{g} / \mathrm{mL}, 100 \mu \mathrm{g} / \mathrm{mL}$, and $200 \mu \mathrm{g} / \mathrm{mL}$. The culture plates were placed in an incubator, and the supernatant was aspirated after $24 \mathrm{~h}$ and then added to $100 \mu \mathrm{L}$ of the medium containing MTT $(0.5 \mathrm{mg} / \mathrm{ml})$ for $4 \mathrm{~h}$. Next, the supernatant was aspirated, and $150 \mu \mathrm{L}$ DMSO was added. The solution was shaken for $10 \mathrm{~min}$ in each well. Finally, the absorbance (OD value) was measured at a wavelength of $490 \mathrm{~nm}$, and the cell survival rate was calculated. 


\subsection{Pharmacodynamics Study}

2.4.1. Grouping. The blank group (K) was grown in $10 \% \mathrm{FBS}$ complete medium. The model group $(M)$ contained $10 \mathrm{ng} / \mathrm{mL}$ TNF- $\alpha$ in the $10 \%$ FBS complete medium. The 12 model experimental groups include: 4 peimine concentrations $(12.5 \mu \mathrm{g} / \mathrm{mL}, 25.0 \mu \mathrm{g} / \mathrm{mL}, 50.0 \mu \mathrm{g} / \mathrm{mL}$, and $100.0 \mu \mathrm{g} / \mathrm{mL}) ; 4$ peiminine concentrations $(12.5 \mu \mathrm{g} / \mathrm{mL}$, $25.0 \mu \mathrm{g} / \mathrm{mL}, \quad 50.0 \mu \mathrm{g} / \mathrm{mL}$, and $100.0 \mu \mathrm{g} / \mathrm{mL}) ; 4$ peimine + peiminine concentrations (peimine : peiminine $=1: 1$, peimine: $12.5,25.0,50.0$, and $100.0 \mu \mathrm{g} / \mathrm{mL}$; peiminine: 12.5 , 25.0, 50.0, and $100.0 \mu \mathrm{g} / \mathrm{mL}$ ).

2.4.2. Index Detection. The A549 cells were seeded at a density of $2 \times 10^{6}$ cells per well in a 6 -well culture plate at $2 \mathrm{~mL}$ and then administered $24 \mathrm{~h}$ later. The supernatant culture solution was collected by centrifugation $48 \mathrm{~h}$ after dosing and then stored at $-80^{\circ} \mathrm{C}$. The cells were then lysed by adding $300 \mu \mathrm{L}$ of RIPA cell lysate per well. The lysate was collected $10 \mathrm{~min}$ later. The supernatant was collected after centrifugation of the lysate, and proteins were detected using the BCA method. The contents of IL-8, MMP-9, and TIMP-1 in the supernatant were determined using an ELISA kit according to the manufacturer's instructions.

\subsection{Cellular Uptake Study}

2.5.1. Apparatus and Operation Conditions. An Ultimate3000 HPLC system (Thermo Scientific, San Jose, USA) equipped with a quaternary pump, an online degasser, an autosampler, a column temperature compartment, and a UV detector was utilized for LC separation. An XBridgeTMC 18 $(2.1 \mathrm{~mm} \times 150 \mathrm{~mm}, 5 \mu \mathrm{m})$ was employed at $30^{\circ} \mathrm{C}$. The mobile phase consisted of acetonitrile (A) and $10 \mathrm{mmol} / \mathrm{L}$ ammonium formate $(\mathrm{B} ; 30: 70)$ that was delivered at a flow rate of $0.3 \mathrm{~mL} / \mathrm{min}$.

The Q Exactive mass spectrometer (Thermo Scientific, San Jose, USA) equipped with a heat electrospray ionization source operated in the full scan mode was used for MS. The carrier gas was nitrogen, and the pressure of the sheath gas and the auxiliary gas were 35 bar and 10 bar, respectively. Positive and negative ions were scanned simultaneously, and the full scan range was from 150 to $1500 \mathrm{~m} / z$, with the firstorder resolution of 70,000 . The spray voltage was $+3.5 \mathrm{kV}$ or $-2.8 \mathrm{kV}$ under the positive or negative mode, respectively. The auxiliary gas heater and capillary temperatures were maintained at $200^{\circ} \mathrm{C}$ and $350^{\circ} \mathrm{C}$, respectively. The ions to be measured and labeled for quantitative analysis were peimine, $\mathrm{m} / z 432.3472[M+H]+$, peiminine, $m / z 430.3316[M+H]+$, and carbamazepine, $m / z 237.1022[M+H]+$.

2.5.2. Cell Sample Preparation. Briefly, the collected cells were placed in a centrifuge tube and centrifuged for $5 \mathrm{~min}$ at $1 \times 10^{4} \mathrm{r} / \mathrm{min}$. The supernatant was discarded, and $300 \mu \mathrm{L}$ ultrapure water was added to each tube. After mixing, the solutions underwent 5 freeze-thaw cycles $\left(-80^{\circ} \mathrm{C} / 37^{\circ} \mathrm{C}\right)$, followed by ultrasonic treatment for $30 \mathrm{~min}$, and then were centrifuged at $12000 \mathrm{r} / \mathrm{min}$ for $20 \mathrm{~min}$. The supernatant was placed into new centrifuge tubes at $2.5 \mu \mathrm{L}$ each to determine protein concentrations via the BCA method. The remaining supernatant was added to $100 \mu \mathrm{l}$ IS and $1200 \mu \mathrm{l}$ methanol [25]. Then, it was shaken for $15 \mathrm{~min}$ to mix thoroughly and centrifuged at $12000 \mathrm{r} / \mathrm{min}$ for $20 \mathrm{~min}$. Next, the supernatant was concentrated via centrifuge, and the residue was dissolved in $100 \mu \mathrm{L}$ of the initial mobile phase to prepare a solution for the experiment. A total of $5 \mu \mathrm{L}$ of the prepared test solution was used for UPLC-MS detection. The final cell uptake was measured by intracellular protein content (drug (ng)/protein ( $\mu \mathrm{g})$ [26], determined three times in parallel with each cell group. The freeze-thaw cycles were repeated at $-80^{\circ} \mathrm{C}$ and $37^{\circ} \mathrm{C}$ and were stored at each temperature for $5 \mathrm{~min}$. The ultrasonic lysis had a power of $250 \mathrm{~W}$ and a frequency of $100 \mathrm{KHz}$, with continuous ultrasound. Three parallel samples were taken at each time point, and each sample was injected three times with an injection volume of $5 \mu \mathrm{L}$, which was calculated using the IS method.

\subsubsection{Validation of the UPLC-MS/MS Method.} Methodological validation conformed to the guidelines for bioanalytical method validation issued by the FDA Center for Drug Evaluation and Research. [27].

(1) Selectivity. The logarithmic phase A549 cells were seeded at a density of $2 \times 106$ cells in a $10 \mathrm{~cm}$ diameter Petri dish and were cultured overnight in a $\mathrm{CO}_{2}$ incubator. Then, cells were divided into two group: the blank group and the administered group. The administered group cells were simultaneously added with peimine and peiminine and then incubated for $240 \mathrm{~min}$ at a final concentration of $100 \mu \mathrm{g} / \mathrm{mL}$. At the end of incubation, the cells were washed four times with PBS. Afterward, trypsin was added to each culture dish to digest the cells, and then the cells in each culture dish were collected and placed in a corresponding centrifuge tube. The intracellular drug concentrations were determined according to the sample preparation method under Section 2.5.2. The cells in each group were measured in parallel three times. The blank cell lysate, cell lysate containing peimine and peiminine, standard peimine and peiminine, and blank methanol were subjected to LC-MS detection according to Section 2.5.1.

(2) Linearity and LOQ. The cells were collected, and a series of cell samples with different concentration were prepared by adding a series of mixed control solutions of $100 \mu \mathrm{L}$ peimine and peiminine and $100 \mu \mathrm{L}$ internal standard carbamazepine reference solution $(249.6 \mathrm{ng} / \mathrm{mL})$ using the sample treatment method under Section 2.5.2. According to the HPLC-MS operation conditions under Section 2.5.1, the concentration of the measured substance in the cell $(\mathrm{ng} / \mathrm{mL})$ was used as the horizontal coordinate. The actual peak area ratio of the measured substance in the cell and the IS was used as the vertical coordinates. The weight of $1 / X$ was taken as the linear regression calculation to obtain the regression equation to establish the standard curve. The minimum detection limit (LOD) was measured with a signal-to-noise ratio $(S / N)$ of 3 : 1 , and the minimum quantitative limit (LOQ) was measured with the signal-to-noise ratio $(S / N)$ of $10: 1$. 
(3) Extraction Recovery and Matrix Effect. The extraction recovery and matrix effect were assessed with six replicates of QC samples at three levels. The extraction recovery was calculated by comparing the mean peak areas of the blank cell lysate with the analytes spiked before and after extraction. The matrix effect was evaluated as the ratio of the peak areas obtained from the cell lysate samples spiked with analysts after extraction with the standard solutions at the corresponding concentration.

(4) Accuracy and Precision. The intra- and interday accuracy and precision were assayed by analyzing six replicates of QC samples at three concentration levels (low, medium, and high) on the same day and on three consecutive days. The relative error and relative standard deviation (RSD) were calculated.

(5) Stability. Six replicate QC samples prepared in parallel at high, medium, and low concentration levels were exposed to various conditions to study sample stability. The short-term stability was carried out by storing the QC samples in the autosampler $\left(4^{\circ} \mathrm{C}\right)$ for $24 \mathrm{~h}$. The long-term stability was performed by storing the QC samples at $-80^{\circ} \mathrm{C}$ for 30 days. For freeze-thaw stability, the $\mathrm{QC}$ samples underwent three complete freeze/thaw cycles at $-20^{\circ} \mathrm{C}-25^{\circ} \mathrm{C}$.

2.5.4. Incubation Time and Uptake. The logarithmic phase A549 cells were seeded at a density of $2 \times 10^{6}$ cells in a $10 \mathrm{~cm}$ diameter Petri dish at $37^{\circ} \mathrm{C}$ in a $5 \% \mathrm{CO}_{2}$ incubator and then cultured for $24 \mathrm{~h}$. Afterward, the supernatant was removed, and the cells were assigned to the following groups: normal peimine group, normal peiminine group, normal combination group, model peimine group, model peiminine group, and model combination group. The peimine and peimine concentrations were $100 \mu \mathrm{g} / \mathrm{mL}$. The action time of the drug was $10,30,45,60$, $90,120,180,240,360$, and $480 \mathrm{~min}$. The drug was removed at the end of the corresponding action times. After incubation, the cells were washed four times with PBS solution in the plate. The cells in each well were collected and placed in a centrifuge tube after trypsin digestion, and the samples were prepared using the method under Section 2.5.2.

2.5.5. Release Assay. The logarithmic phase A549 cells were seeded at a density of $2 \times 10^{6}$ cells in a $10 \mathrm{~cm}$ diameter Petri dish and cultured at $37^{\circ} \mathrm{C}$ in a $5 \% \mathrm{CO}_{2}$ incubator for $24 \mathrm{~h}$. Afterward, the supernatant was removed. For drug release measurements, a certain concentration of peimine and peiminine was spiked to make the final concentration of the drug $100 \mu \mathrm{g} / \mathrm{mL}$ in the peimine and peiminine administration groups and then incubated for $120 \mathrm{~min}$ and $60 \mathrm{~min}$, respectively. Meanwhile, a certain concentration of mixed solution was added to make the final concentrations of peimine and peimine in the combined administration groups $100 \mu \mathrm{g} / \mathrm{mL}$ and then incubated for $240 \mathrm{~min}$. At the end of incubation, the cells were washed four times with PBS and then incubated for $0,10,30,45,60,90,120,180,240$, $360,480,720$, and $1440 \mathrm{~min}$ in a fresh culture medium and placed in the incubator. Afterward, the cells were washed four times with PBS solution. Then, trypsin was added to each culture dish to digest the cells. Finally, the cells in each culture dish were collected and placed in a corresponding centrifuge tube. The cellular drug concentrations were determined according to the method under Section 2.5.2. Each group was measured in parallel three times.

2.6. Statistical Methods. Statistical analysis was calculated using SPSS19.0 software. Mapping analysis was performed using GraphPad Prism 7.00 drawing software. The values were expressed in terms of mean \pm standard deviation. Differences were compared between the groups using oneway ANOVA. $P$ value $<0.05$ indicated statistically significant differences. $P$ values $<0.01$ were considered significant.

\section{Results}

3.1. Cell Viability Assays. As shown in Figure 1, there were significant differences in cell viability between the model blank cell group and the normal blank cell group $(P<0.01)$, indicating that the A549 cell model was established successfully. Compared to the model blank group, the model single group and the model combination group showed a significant increase in cell viability with an increasing trend when the concentrations of the peimine and peiminine were $25-100 \mu \mathrm{g} / \mathrm{mL}(P<0.05)$.

3.2. Pharmacodynamic Results. The interleukin-8 (IL-8), matrix metalloprotein-9 (MMP-9), and tissue inhibitor of metalloproteinases-1 (TIMP-1) in the blank group, the model group, and the 12 experimental groups are summarized in Table 1 and Figure 2. Compared to the blank group, the inflammatory cytokines (IL-8, MMP-9, and TIMP-1) were significantly increased, whereas TIMP-1/ MMP-9 was significantly decreased $(P<0.01)$ in the model group. These results showed that the A549 cellular inflammatory model was established successfully and that the pharmacodynamic characteristics could be reflected with the three inflammatory cytokines. Meanwhile, IL-8 was significantly decreased in the $50.0 \mu \mathrm{g} / \mathrm{mL}$ combination and the $100.0 \mu \mathrm{g} / \mathrm{mL}$ combination groups. In addition, the highest efficacy was seen in the $100.0 \mu \mathrm{g} / \mathrm{mL}$ combination group, which showed a significant reduction in the three inflammatory cytokines and an increase in TIMP-1/MMP-9.

\subsection{Cellular Uptake Studies}

3.3.1. Screen Optimal Dosing Concentration by MTT Colorimetry. The final concentration of peimine and peiminine in the cellular pharmacokinetic experiment was $100 \mu \mathrm{g} / \mathrm{mL}$ according to the cell viability assay and pharmacodynamic results (Figure 3).

\subsubsection{Validation of the UPLC-MS/MS Method}

(1) Selectivity. The retention time of the drug-containing cell lysates was consistent with that of standard substances. There were no interference peaks in the blank cell lysates to peimine and peiminine, and high responsiveness and good separation were achieved. 


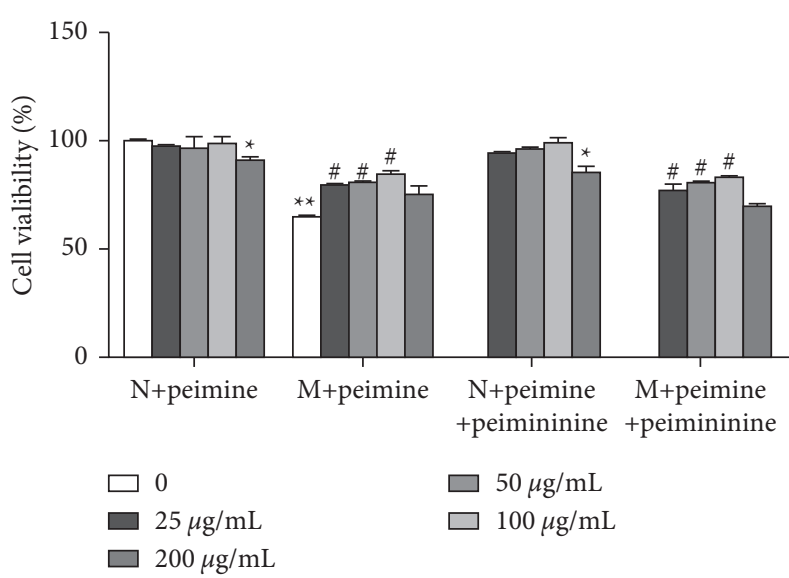

(a)

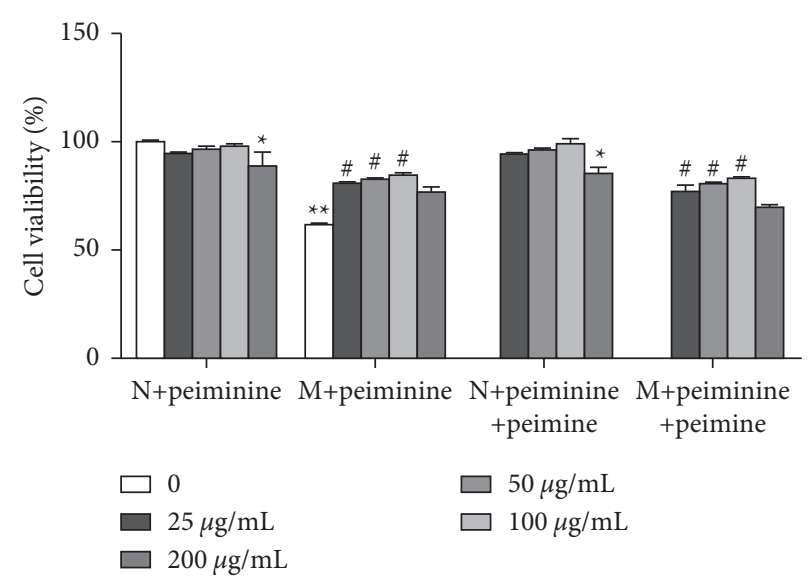

(b)

FIGURE 1: Effect of different concentrations of peimine and peiminine on the viability of A549 cells. (a): $N+$ peimine: normal group $(0 \mu \mathrm{g} / \mathrm{mL})$, normal single groups $(25,50,100$, and $200 \mu \mathrm{g} / \mathrm{mL}) ; M+$ peimine: model group $(0 \mu \mathrm{g} / \mathrm{mL})$, model single groups $(25,50,100$, and $200 \mu \mathrm{g} / \mathrm{mL}) ; N+$ peimine + peiminine: normal combination groups $(25,50,100,200 \mu \mathrm{g} / \mathrm{mL} ; M+$ peimine + peiminine: model combination groups $(25,50,100$, and $200 \mu \mathrm{g} / \mathrm{mL})$. (b): $N$ + peiminine: normal group $(0 \mu \mathrm{g} / \mathrm{mL})$, normal single groups $(25,50,100,200 \mu \mathrm{g} / \mathrm{mL})$; $M+$ peiminine: model group $(0 \mu \mathrm{g} / \mathrm{mL})$, model single groups $(25,50,100$, and $200 \mu \mathrm{g} / \mathrm{mL}) ; N+$ peiminine + peimine: normal combination groups $(25,50,100$, and $200 \mu \mathrm{g} / \mathrm{mL}) ; M+$ peiminine + peimine: model combination groups $(25,50,100$, and $200 \mu \mathrm{g} / \mathrm{mL})$. The concentrations of peimine and peiminine in the combination groups were as follows: peimine:peiminine $=1: 1$, peimine: $25,50,100$, and $200 \mu \mathrm{g} / \mathrm{mL}$ and peiminine: $25,50,100$, and $200 \mu \mathrm{g} / \mathrm{mL}\left({ }^{*} P<0.05\right.$ and ${ }^{* *} P<0.01$ mean significant difference compared with the normal group, and ${ }^{\#} P<0.05$ means significant difference compared with the model group).

(2) Linearity and LOQ. The linear ranges of peimine and peiminine were $0.4008-2004 \mathrm{ng} / \mathrm{mL}$ and $0.2840-1420 \mathrm{ng} / \mathrm{mL}$, respectively. The correlation coefficient $(r)$ was greater than 0.9970. The minimum detection limits for peimine and peiminine were 0.1982 and $0.0951 \mathrm{ng} / \mathrm{mL}$, respectively, which ensured the accurate determination of low concentrations in the cell samples (Table 2).

(3) Extraction Recovery and Matrix Effect. The matrix effects were $95.05 \%-111.29 \%$, indicating that the cell matrix did not affect the quantitative detection of peimine and peiminine. The extraction recovery rates were $83.85 \%-113.67 \%$, indicating that the experimental method was good (Table 3 ).

(4) Accuracy and Precision. As shown in Table 4, the intraand interday precision of peimine and peiminine were $2.14 \%-6.73 \%$ and $4.33 \%-7.73 \%$. The intra- and interday accuracy were $-1.29 \%-1.76 \%$ and $-0.22 \%-3.05 \%$, thus satisfying the requirements.

(5) Stability. Table 5 summarizes the stability evaluation results of the analysts under the different storage conditions. The measured concentration deviations of the quality control samples were less than $\pm 15 \%$ of its nominal values after treatment under different conditions. This indicates that the method had satisfactory stability for the determination of peimine and peiminine and that it was suitable for sample analysis.

3.3.3. Incubation Time and Uptake. The relationship between incubation time and the cellular uptake of peimine and peiminine was quantified by measuring the cellular concentrations at various time points after cell lysis, as presented in Table 6. As shown in Figure 4, the uptake characteristics of peimine and peiminine in the A549 cells were significantly different. In the single group, the peak times $\left(T_{\max }\right)$ of the cell uptake of peimine and peiminine were $120 \mathrm{~min}$ and $60 \mathrm{~min}$, respectively. In the combination group, $T_{\max }$ of peimine and peiminine were $240 \mathrm{~min}$. In the combination group, the maximum intake $\left(I_{\max }\right)$ of peimine and peiminine was significantly higher than in the single administration group. $I_{\max }$ of peimine and peiminine was higher in the model combination group than the normal combination group.

3.3.4. Release Assay. As presented in Table 7, the relationship between the release time and intracellular uptake of peimine and peiminine was assessed using the intracellular drug concentrations after cell lysis. The calculated mean intracellular drug concentrations of peimine and peiminine were plotted as different times after withdrawal for each group. In the normal (single) group, model (single) group, normal (combination) group, and model (combination) group, both peimine and peiminine were rapidly released in the cells.

\section{Discussion}

The precipitation protein method (PPT) was selected for sample pretreatment in this study, because PPT is economical, practical, and simple to perform [28]. At present, the commonly used reagents for protein removal are methanol, acetonitrile, chloroform, and ethyl acetate. In this experiment, the matrix effect and extraction recovery rates of peimine, peiminine, and IS were investigated under methanol and 


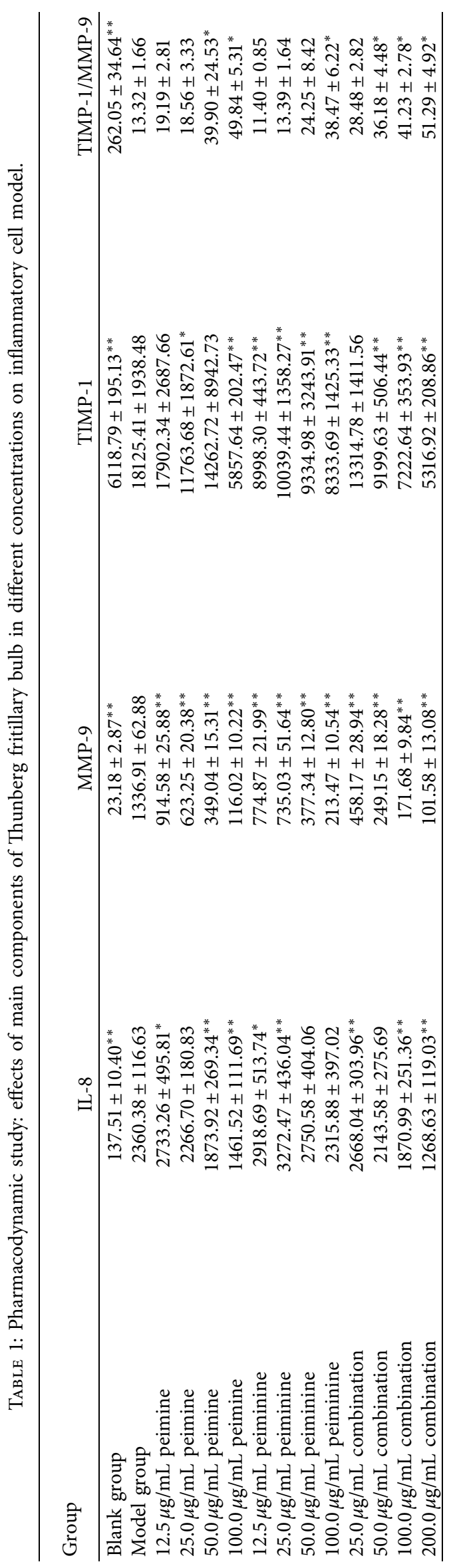




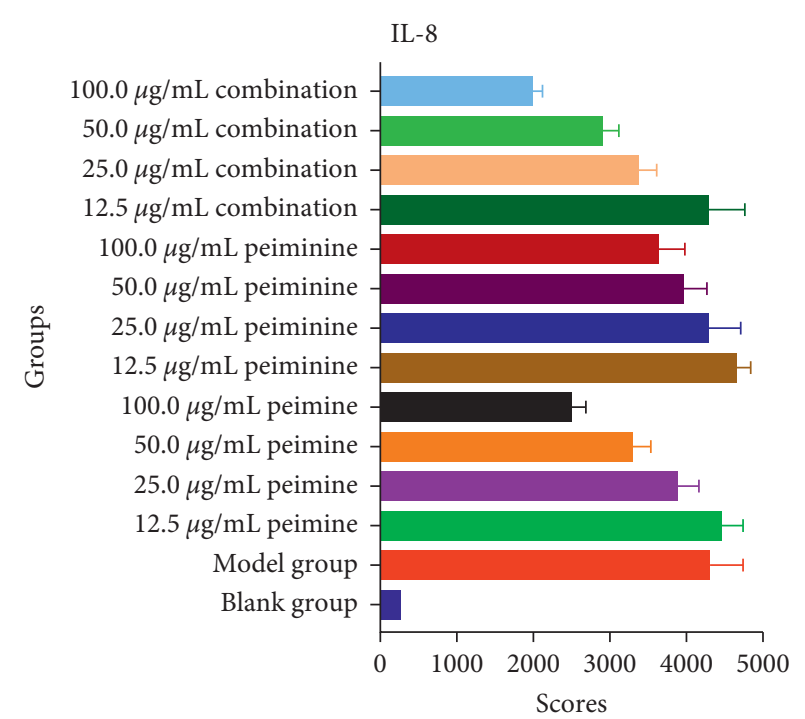

(a)

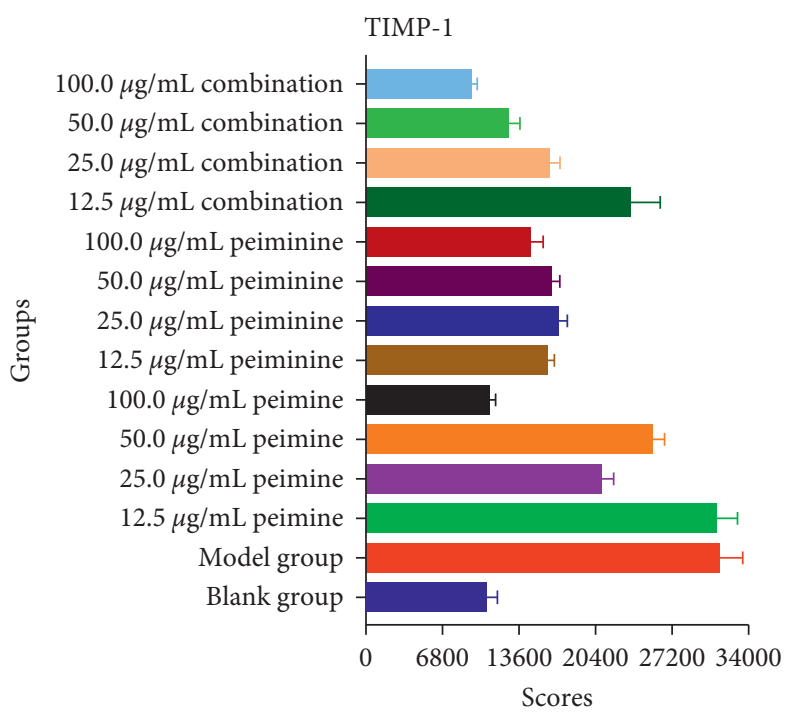

(c)

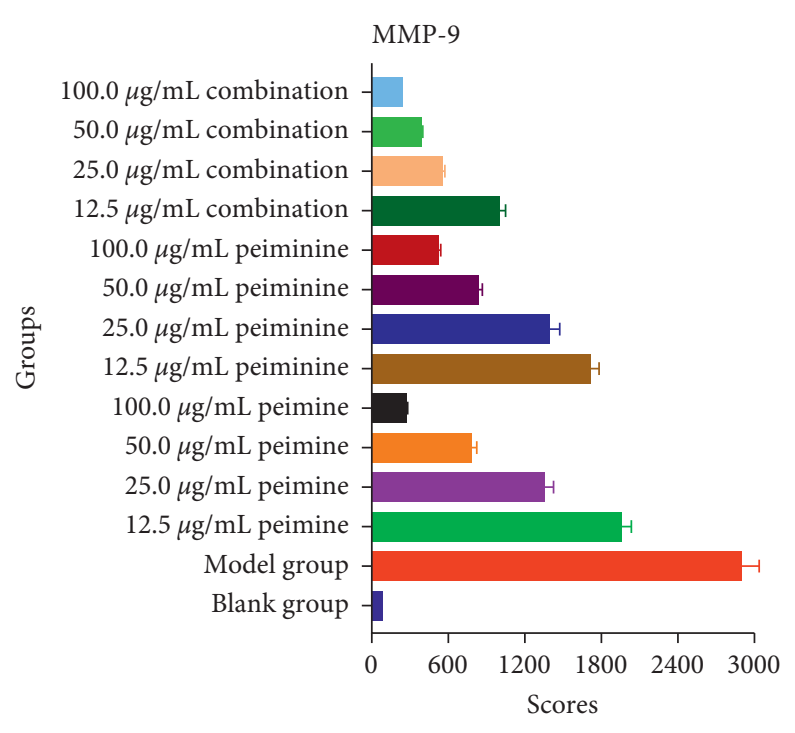

(b)

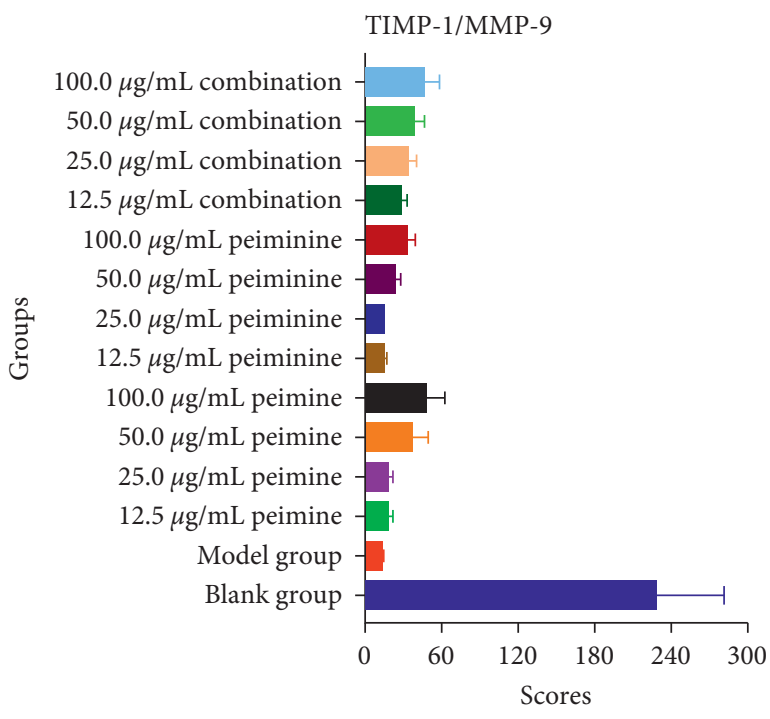

(d)

Figure 2: Inflammatory cytokines: (a) IL-8, (b) MMP-9, (c) TIMP-1, and (d) TIMP-1/MMP-9.

acetonitrile $(1: 1)$ and cell dosage $4: 1$. The results showed that pure methanol could remove the proteins with less interference than the other two solvents and that the matrix effects were $75 \%-115 \%$, with recovery rates of greater than $70 \%$. In the evaluation of the 4 mobile phase systems, methanol water (A), acetonitrile water (B), acetonitrile $0.1 \%$ formic acid water $(\mathrm{C})$, and acetonitrile- $10 \mathrm{mmol} / \mathrm{mL}$ ammonium formate (D), it was shown that the method selected (D) to determine the peimine and peiminine and to separate the components to be tested and IS was good. The interference of the matrix was lower.

Inflammation is the body's natural defense response to various injury factors and the common pathological process in various diseases, which involves multiple mechanisms and pathways. Matrix metalloproteinase inhibitors (TIMPs) are endogenous natural inhibitors of MMPs, which can form complexes (1:1 binding) with MMPs to inhibit them [29].
Thus, three inflammatory cytokines and TIMP-1/MMP-9 were considered as a whole. The anti-inflammatory effects of the $100.0 \mu \mathrm{g} / \mathrm{mL}$ combination group were the most obvious of the 12 experimental groups (删除, 改为: compared with the model group, the MMP-9 and TIMP-1 of peimine and peiminine of the $100.0 \mu \mathrm{g} / \mathrm{mL}$ showed significant difference $(P<0.01)$. There are significant differences of IL-8, MMP-9, and TIMP-1 between the model group and the combination group $(P<0.01)$. Interestingly, the anti-inflammatory effects are more attributed to peimine than peiminine). Peimine showed a dose-dependent inhibition of MMP-9 secretion in the concentration range of $12.5-100 \mu \mathrm{g} / \mathrm{mL}$ and significantly decreased IL-8 secretion at concentrations of $50 \mu \mathrm{g} / \mathrm{mL}$ and $100 \mu \mathrm{g} / \mathrm{mL}$. TIMP-1 secretion of peimine was significantly decreased at concentrations of $100 \mu \mathrm{g} / \mathrm{mL}$, while the ratio of TIMP-1/MMP-9 was significantly increased at $100 \mu \mathrm{g} / \mathrm{mL}$. 

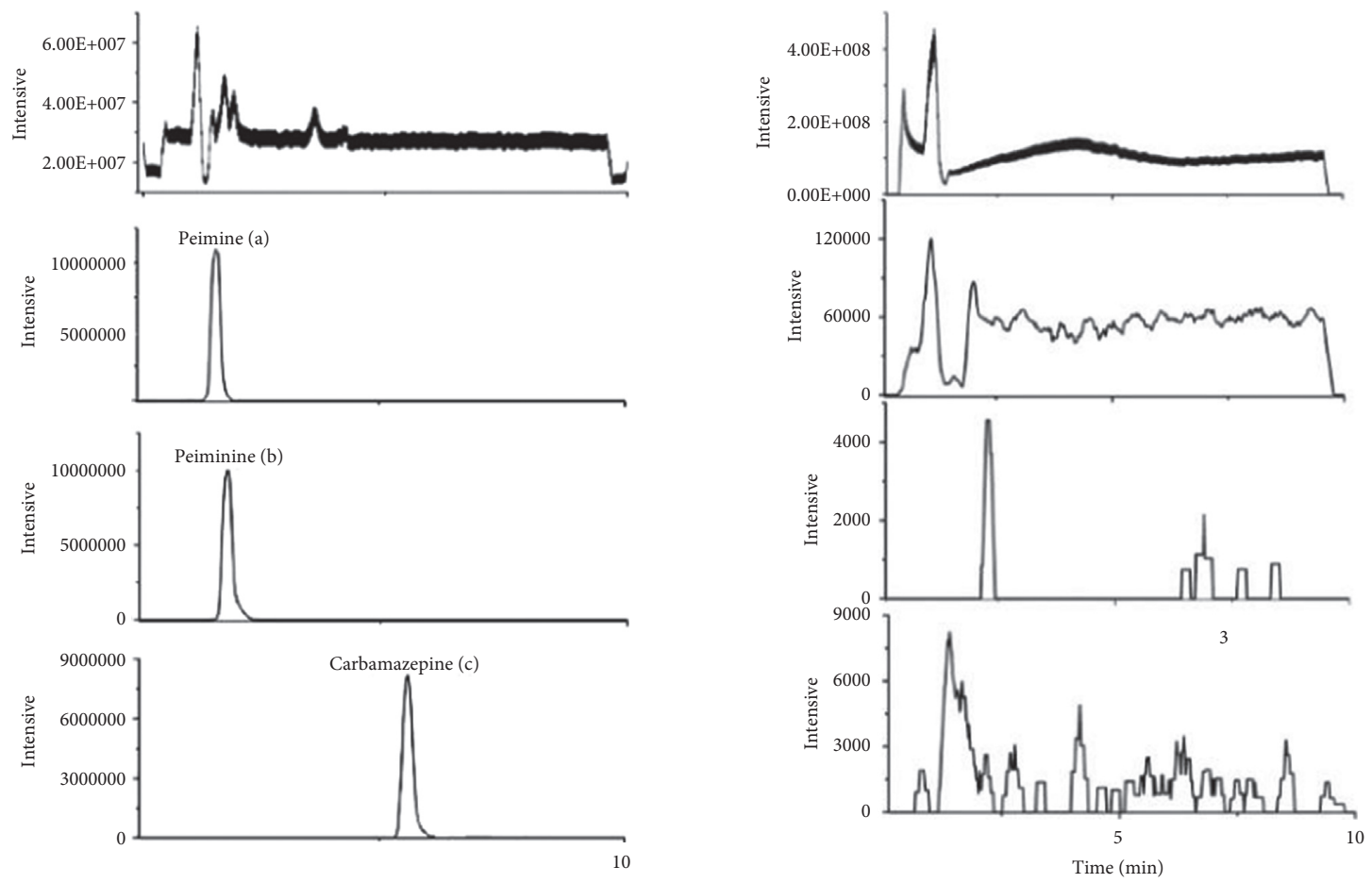

(a)

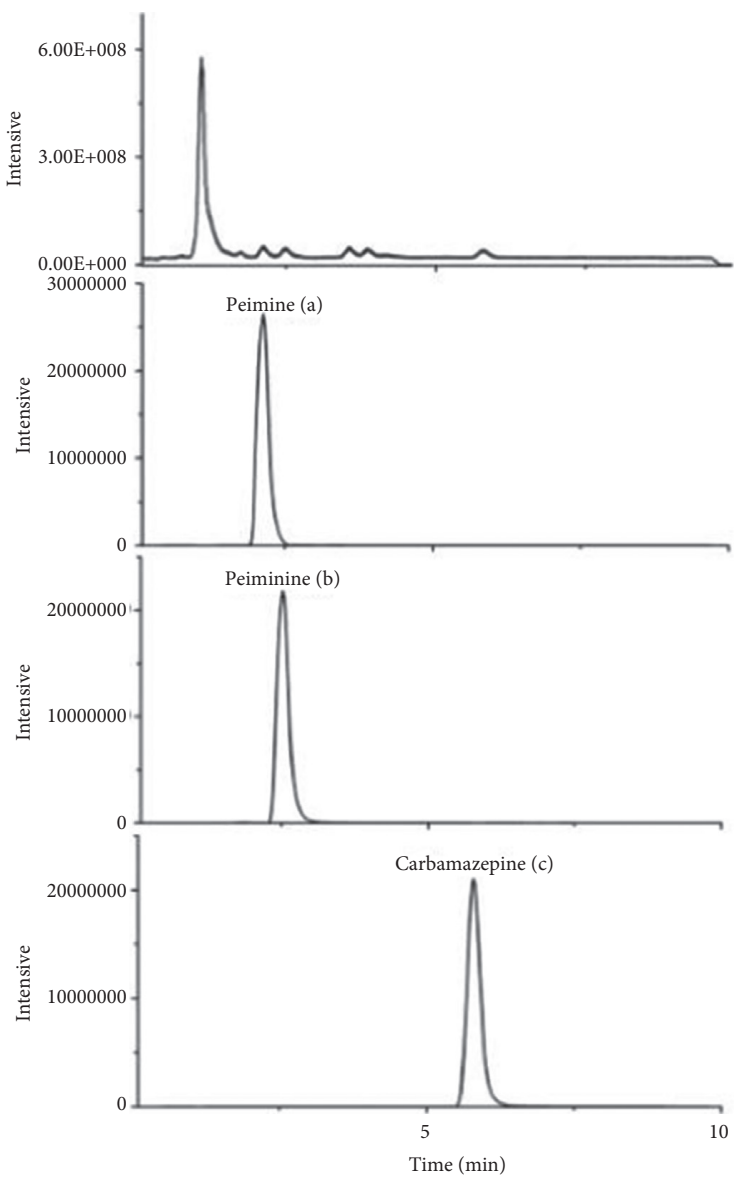

(b)

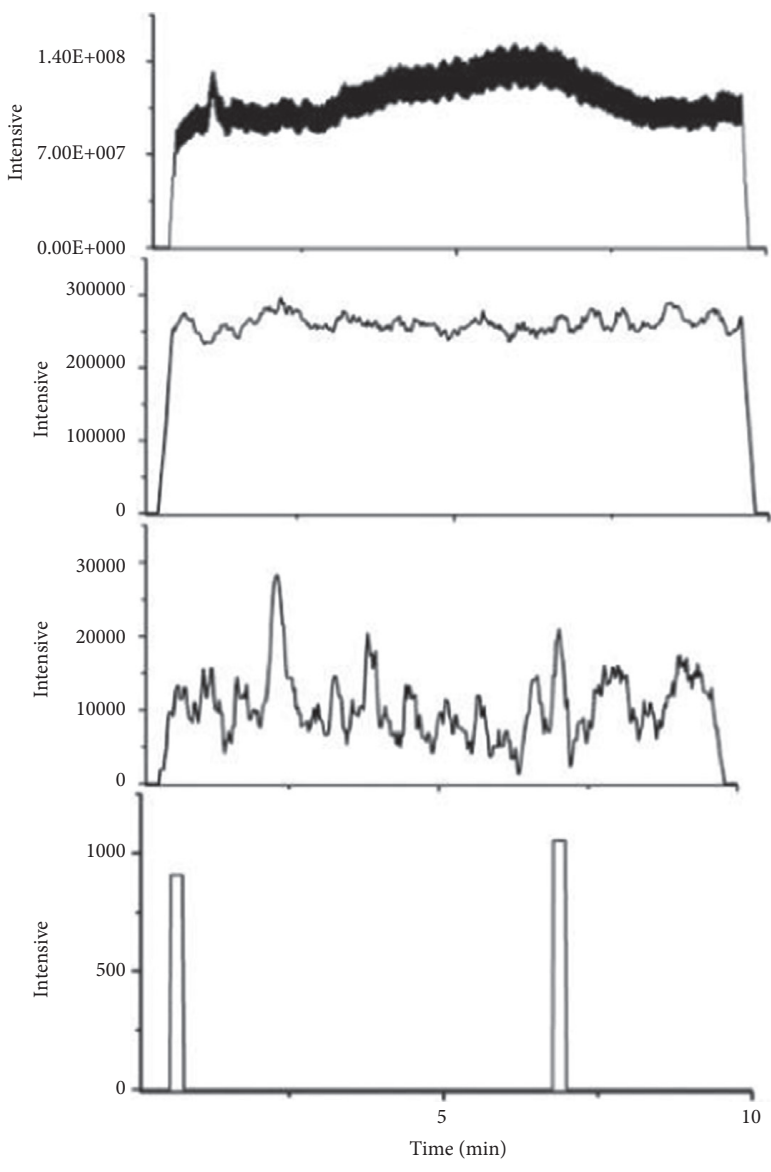

(c)

(d)

FIGURE 3: Representative chromatograms of peimine (a), peiminine (b), and IS (c): (a) chromatogram of mixed reference; (b) chromatogram of blank methanol; (c) chromatogram of cell extracts containing drugs; (d) chromatogram of blank cell. 
TABLE 2: Regression equation, linearity, and LOQ.

\begin{tabular}{lccccc}
\hline Component & Regression equation & Linear range $(\mathrm{ng} / \mathrm{mL})$ & LOQ $(\mathrm{ng} / \mathrm{mL})$ & LOD $(\mathrm{ng} / \mathrm{mL})$ & Correlation coefficient $(r)$ \\
\hline Peimine & $y=0.0073 X+0.017$ & $0.4008 \sim 2004$ & 0.4008 & 0.1982 & 0.9979 \\
Peiminine & $y=0.0082 X+0.007$ & $0.2840 \sim 1420$ & 0.2840 & 0.0951 & 0.9999 \\
\hline
\end{tabular}

TABle 3: Extraction recovery and matrix effect at low, medium, and high concentration levels (mean $\pm \mathrm{SD}, n=6$ ).

\begin{tabular}{|c|c|c|c|c|c|}
\hline \multirow{2}{*}{ Component } & \multirow{2}{*}{ Spiked (ng/mL) } & \multicolumn{2}{|c|}{ Recovery } & \multicolumn{2}{|c|}{ Matrix effect } \\
\hline & & Mean (\%) & RSD (\%) & Mean (\%) & RSD (\%) \\
\hline \multirow{3}{*}{ Peimine } & 2.004 & $89.81 \pm 0.80$ & 0.89 & $106.55 \pm 0.26$ & 0.24 \\
\hline & 20.04 & $102.04 \pm 3.07$ & 3.01 & $95.05 \pm 2.20$ & 2.31 \\
\hline & 200.4 & $83.85 \pm 2.86$ & 3.41 & $109.25 \pm 1.76$ & 1.61 \\
\hline \multirow{3}{*}{ Peiminine } & 1.42 & $113.67 \pm 2.32$ & 2.04 & $111.29 \pm 1.80$ & 1.62 \\
\hline & 14.2 & $84.89 \pm 3.54$ & 4.17 & $96.31 \pm 4.96$ & 5.15 \\
\hline & 142 & $92.04 \pm 4.49$ & 4.88 & $95.44 \pm 4.79$ & 5.02 \\
\hline
\end{tabular}

TABLE 4: Intraday and interday accuracy and precision.

\begin{tabular}{lccccccc}
\hline Component & $\begin{array}{c}\text { Spiked } \\
(\mathrm{ng} / \mathrm{mL})\end{array}$ & $\begin{array}{c}\text { Measured } \\
(\mathrm{ng} / \mathrm{mL})\end{array}$ & $\begin{array}{c}\text { Intraday } \\
\text { Accuracy } \\
(\mathrm{RE})\end{array}$ & $\begin{array}{c}\text { Precision } \\
(\mathrm{RSD} \%)\end{array}$ & $\begin{array}{c}\text { Measured } \\
(\mathrm{ng} / \mathrm{mL})\end{array}$ & $\begin{array}{c}\text { Interday } \\
\text { Accuracy } \\
(\mathrm{RE})\end{array}$ & $\begin{array}{c}\text { Precision } \\
(\mathrm{RSD})\end{array}$ \\
\hline \multirow{3}{*}{ Peimine } & 2.004 & $2.02 \pm 0.13$ & 0.55 & 6.24 & $2.01 \pm 0.08$ & 0.38 & 4.33 \\
& 20.04 & $20.02 \pm 0.81$ & -0.08 & 4.05 & $20.28 \pm 1.25$ & 1.19 & 6.19 \\
\hline \multirow{3}{*}{ Peiminine } & 200.4 & $199.87 \pm 4.28$ & -0.26 & 2.14 & $199.96 \pm 9.87$ & -0.22 & 4.94 \\
& 1.42 & $1.45 \pm 0.06$ & 1.76 & 3.91 & $1.46 \pm 0.11$ & 3.05 & 7.73 \\
& 14.2 & $14.39 \pm 0.96$ & 1.34 & 6.73 & $14.58 \pm 0.53$ & 2.69 & 3.63 \\
\end{tabular}

TABLE 5: Stability investigated for peimine and peiminine under different conditions.

\begin{tabular}{|c|c|c|c|c|c|c|c|}
\hline \multirow{2}{*}{ Component } & \multirow{2}{*}{ Spiked (ng/mL) } & \multicolumn{2}{|c|}{ Short-time stability } & \multicolumn{2}{|c|}{ Long-time stability } & \multicolumn{2}{|c|}{ Freeze-thaw (three cycles) } \\
\hline & & Measured $(\mathrm{ng} / \mathrm{mL})$ & Accuracy (\%) & Measured (ng/mL) & Accuracy (\%) & Measured $(\mathrm{ng} / \mathrm{mL})$ & Accuracy (\%) \\
\hline \multirow{3}{*}{ Peimine } & 2.004 & $2.06 \pm 0.13$ & 2.96 & $2.07 \pm 0.09$ & 3.46 & $1.99 \pm 0.17$ & -0.69 \\
\hline & 20.04 & $20.55 \pm 1.17$ & 2.52 & $20.39 \pm 0.92$ & 1.74 & $20.62 \pm 1.37$ & 2.89 \\
\hline & 200.4 & $200.81 \pm 1.31$ & 0.21 & $200.69 \pm 2.84$ & 0.15 & $200.84 \pm 3.22$ & 0.22 \\
\hline \multirow{3}{*}{ Peiminine } & 1.42 & $1.46 \pm 0.07$ & 2.46 & $1.44 \pm 0.07$ & 1.29 & $1.43 \pm 0.06$ & 0.94 \\
\hline & 14.2 & $14.52 \pm 0.57$ & 2.27 & $14.42 \pm 0.79$ & 1.57 & $14.53 \pm 0.49$ & 2.35 \\
\hline & 142 & $144.12 \pm 5.57$ & 1.49 & $143.02 \pm 3.70$ & 0.71 & $141.19 \pm 2.97$ & -0.57 \\
\hline
\end{tabular}

In addition, peiminine also inhibited the secretion of MMP9 in a dose-dependent manner and significantly inhibited the secretion of TIMP-1 in a concentration range of $12.5-100 \mu \mathrm{g} / \mathrm{ml}$. At the doses of $25 \mu \mathrm{g} / \mathrm{mL}$ and $50 \mu \mathrm{g} / \mathrm{mL}$, the amount of TIMP-1 secreted did not change significantly, indicating that the inhibitory effect of peiminine was at a plateau stage, which differed from previous studies [30].

Considering the cellular uptake of peimine and peiminine, their final concentrations of $100 \mu \mathrm{g} / \mathrm{mL}$ were screened using the MTT colorimetric method and combined with the pharmacodynamic results. Next, the relationship between incubation time and peimine and peiminine uptake was investigated. The results showed that the uptake of peimine increased with prolonged incubation time within $120 \mathrm{~min}$ in the single groups. Among them, the normal group showed that the uptake of peimine started to increase rapidly at $90 \mathrm{~min}$ and then decreased slowly after $120 \mathrm{~min}$. In the range of 180-480 min, a steady state was reached. The model group showed that the uptake of peimine began to increase rapidly at $45 \mathrm{~min}$, with a change range of $240-480 \mathrm{~min}$ that was not significant, and reached a steady state. In the combination group, the uptake of peimine increased with the prolonged incubation time within $240 \mathrm{~min}$. In the single group, $T_{\max }$ of peiminine was $60 \mathrm{~min}$, whereas the combination group had a peiminine $T_{\max }$ that was the same as that of peimine at $240 \mathrm{~min}$. In the normal single group and the model single group, $I_{\max }$ of peimine was $1667.58 \pm 109.83$ and $1823.28 \pm 97.18 \mathrm{ng} / \mu \mathrm{g}$, respectively, with no statistical difference $(P>0.05)$. $I_{\max }$ of peiminine was $449.07 \pm 39.65$ and $622.72 \pm 72.70 \mathrm{ng} / \mu \mathrm{g}$, with significant differences $(P<0.05 P<0.05) . I_{\max }$ of peimine and peiminine in the normal combination group were $2933.14 \pm 126.76$ and $765.37 \pm 99.30 \mathrm{ng} / \mu \mathrm{g}$, respectively, which 


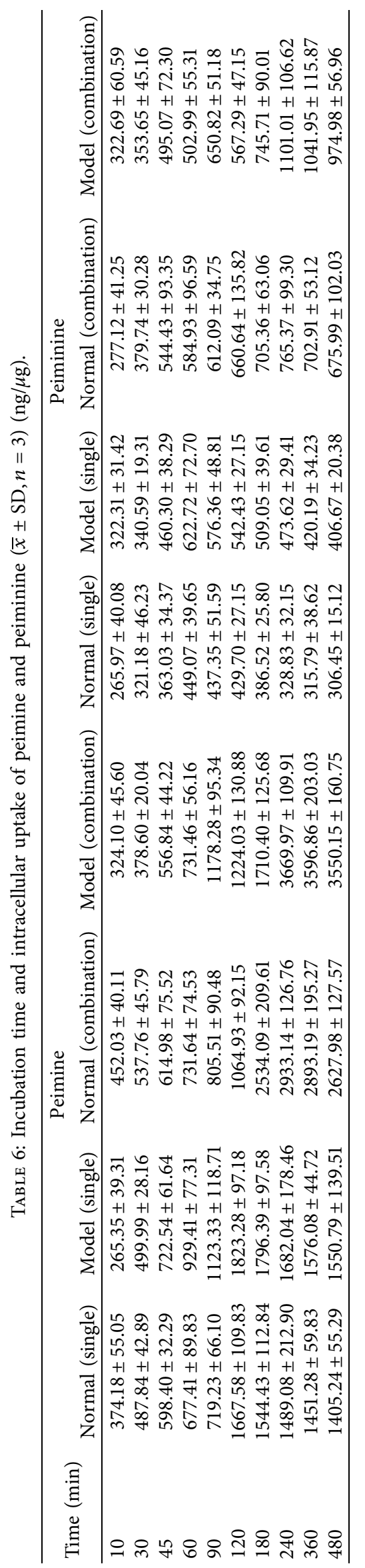




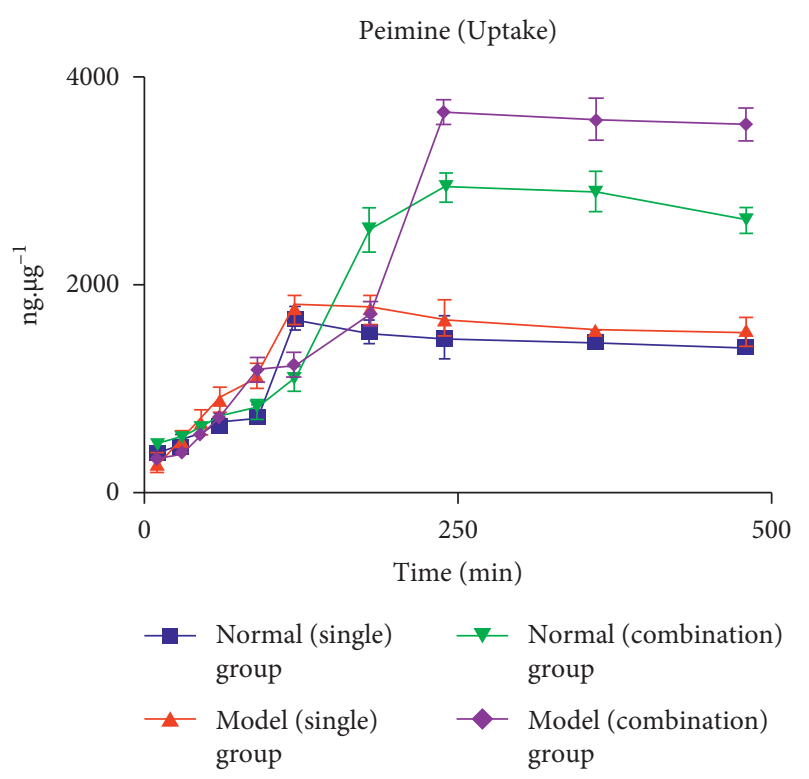

(a)

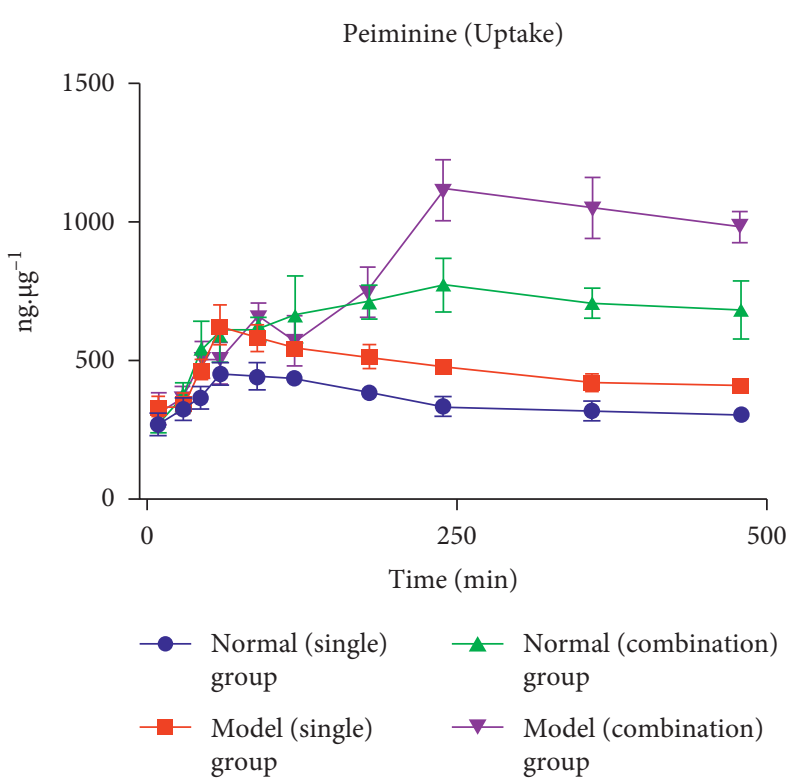

(b)

FIGURE 4: Incubation time and uptake curves (mean $\pm \mathrm{SD}, n=3$ ): (a) peimine; (b) peiminine

were significantly higher than that of the normal single group $(P<0.01) . T_{\max }$ of peimine and peiminine were prolonged in both the normal and model combination groups, which may be due to the similar structure of peimine and peiminine, indicating that the drug had some competitive effects in the process of entering cells. Compared with the single group, the combination group had a significantly increased uptake of peimine and peiminine, which may be due to the synergistic effect of peimine and peiminine to change some cellular mechanisms and increase cellular drug uptake, but this phenomenon needs further research (删除, 改为: in the model cells, $T_{\max }$ of uptake of peimine and peiminine were $120 \mathrm{~min}$ and $60 \mathrm{~min}$ with the combination group $240 \mathrm{~min}$. $T_{\max }$ were prolonged significantly in combination group $(P<0.01)$. The same trend was seen in normal cells. The reason was speculated that because of the the similar structure of peimine and peiminine, there may be a competitive effect in the process of uptake. In the experiment involving the intracellular release of peimine and peiminine, the normal single group, the model single group, the normal combination group, and the model combination group rapidly eliminated peimine and peiminine in the cells, with a maximum removal rate of $30 \mathrm{~min}$, and the eliminating trend was the same. This may be related to how the drug was discharged intracellularly and the similar structure of peimine and peiminine, indicating that A549 cells had strong release abilities to peimine and peiminine in both normal and model conditions.

Combined with previous pharmacodynamic and uptake studies of peimine and peiminine at the cellular level, this study showed that the pharmacodynamic strength typically relates to its uptake in the cell. In this experiment, the concentrations of peimine and peiminine were $100 \mu \mathrm{g} / \mathrm{mL}$, $I_{\max }$ of peimine being significantly higher than peiminine, and the combination group was significantly increased compared with the single group. This trend was the same for anti-inflammatory effects: $\quad 100.0 \mu \mathrm{g} / \mathrm{mL}$ combination $>100.0 \mu \mathrm{g} / \mathrm{mL}$ peimine $>100.0 \mu \mathrm{g} / \mathrm{mL}$ peiminine. Compared to the normal combination group, $I_{\max }$ of peimine and peiminine increased in the model combination group. This might be due to the significant inhibitory effects of peimine and peiminine on cytokine secretion, thus increasing the uptake of cellular drugs in the model group. 


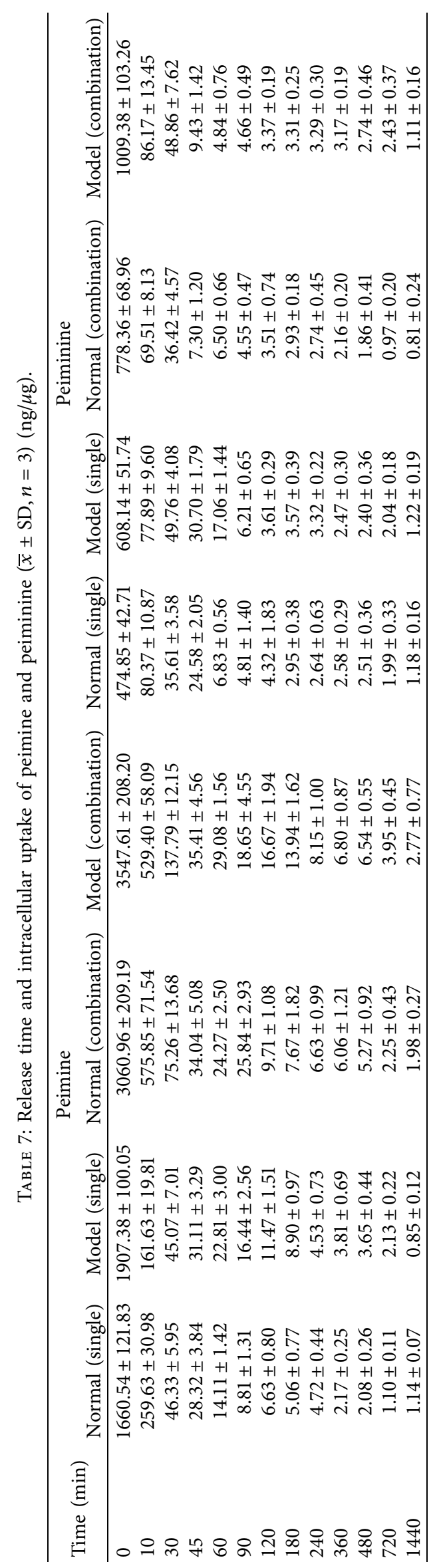




\section{Conclusions}

The UPLC-MS/MS method established in this study was found to be selective and stable for the simultaneous determination of peimine and peiminine in A549 cells. The model group could uptake more than the normal group. Compared with the single group, the combination group showed a higher untake. The combination group's antiinflammatory effects were improved effectively. In this paper, the uptake of peimine and peiminine in cellular was systematically investigated, and the scientific date can provide a basis for the research of anti-inflammatory mechanisms and may be useful for the further clinical practice of of peimine and peiminine.

\section{Data Availability}

The data used to support the findings of this study are included within the article.

\section{Conflicts of Interest}

The authors declare no conflicts of interest.

\section{Acknowledgments}

This research was supported financially by the Henan Scientific and Technological Research Project (no. 202102310173) and Postdoctoral Fund of Henan University of Chinese Medicine (no. BSJJ201601).

\section{References}

[1] H.-J. Li, Y. Jiang, and P. Li, "Chemistry, bioactivity and geographical diversity of steroidal alkaloids from the Liliaceae family," Natural Product Reports, vol. 23, no. 5, pp. 735-752, 2006.

[2] H. Li, A. Hung, M. Li, and A. Yang, "Fritillariae thunbergii Bulbus: traditional uses, phytochemistry, pharmacodynamics, pharmacokinetics and toxicity," International Journal of Molecular Sciences, vol. 20, no. 7, p. 1667, 2019.

[3] L. Qiu, "Study on the efficacy evolution of fritillaria cirrhosa D.don and fritillaria thunbergii miq," Journal of Anhui University of Chinese Medicine, vol. 37, no. 6, pp. 1-3, 2018.

[4] S. H. Nile, J. Su, D. Wu et al., "Fritillaria thunbergii Miq. (Zhe Beimu): a review on its traditional uses, phytochemical profile and pharmacological properties," Food and Chemical Toxicology, vol. 153, Article ID 112289, 2021.

[5] J. Xu, W. Zhao, L. Pan et al., "Peimine, a main active ingredient of Fritillaria, exhibits anti-inflammatory and pain suppression properties at the cellular level," Fitoterapia, vol. 111, pp. 1-6, 2016.

[6] H. Tan, G. Zhang, X. Yang, T. Jing, D. Shen, and X. Wang, "Peimine inhibits the growth and motility of prostate cancer cells and induces apoptosis by disruption of intracellular calcium homeostasis through Ca 2+/CaMKII/JNK pathway," Journal of Cellular Biochemistry, vol. 121, no. 1, pp. 81-92, 2019.

[7] M. F. Zhang and Y. Q. Shen, "Advances in research on pharmacology of Fritillaria," Shanghai Medical \& Pharmacology of Fritillaria, vol. 10, pp. 459-461, 2007.
[8] P.-F. Yi, Y.-C. Wu, H.-B. Dong et al., "Peimine impairs proinflammatory cytokine secretion through the inhibition of the activation of NF- $\kappa$ B and MAPK in LPS-induced RAW264.7 macrophages," Immunopharmacology and Immunotoxicology, vol. 35, no. 5, pp. 567-572, 2013.

[9] G. X. Gui, "An effect of the peimine on TNF- $\alpha$, IL-4, IL-10 and PEG-2 expression of mice with acute lung injury," Clinical Journal of Chinese Medicine, vol. 9, no. 1, pp. 1-4, 2017.

[10] H. Guo, F. Ji, B. Liu et al., "Peiminine ameliorates bleomycininduced acute lung injury in rats," Molecular Medicine Reports, vol. 7, no. 4, pp. 1103-1110, 2013.

[11] Z. Luo, B. Zheng, B. Jiang, X. Xue, E. Xue, and Y. Zhou, "Peiminine inhibits the IL- $1 \beta$ induced inflammatory response in mouse articular chondrocytes and ameliorates murine osteoarthritis," Food \& Function, vol. 10, no. 4, pp. 2198-2208, 2019.

[12] G. Chen, J. Liu, L. Jiang et al., "Peiminine protects dopaminergic neurons from inflammation-induced cell death by inhibiting the ERK1/2 and NF- $\kappa$ B signalling pathways," International Journal of Molecular Sciences, vol. 19, no. 3, p. 821, 2018.

[13] B. Lee, E.-Y. Kim, J.-H. Kim et al., "Antiallergic effects of peiminine through the regulation of inflammatory mediators in HMC-1 cells," Immunopharmacology and Immunotoxicology, vol. 37, no. 4, pp. 351-358, 2015.

[14] P. Ni, J. W. Zhang, and J. L. Liu, "Research progress in cellular pharmacokinetics," Progress in Pharmaceutical Sciences, vol. 38, no. 12, pp. 881-885, 2014.

[15] F. Zhou, J. Zhang, P. Li et al., "Toward a new age of cellular pharmacokinetics in drug discovery," Drug Metabolism Reviews, vol. 43, no. 3, pp. 335-345, 2011.

[16] W. Zha, G. Liang, J. Xiao et al., "Berberine inhibits HIV protease inhibitor-induced inflammatory response by modulating ER stress signaling pathways in murine macrophages," PLoS One, vol. 5, no. 2, p. e9069, 2010.

[17] C. X. Liu, "The role of pharmacokinetics in the research on the conversion of new drugs," Science and Technology Review, vol. 30, no. 5, pp. 67-71, 2012.

[18] N. Zheng, X. Wang, and Y. Q. Wang, "An UPLC-MS/MS method for quantification of a novel doxorubicin conjugation prodrug in tumor cells," Acta Pharmaceutica Sinica, vol. 53, no. 2, pp. 278-283, 2018.

[19] J. Zhang, F. Zhou, X. Wu et al., "Cellular pharmacokinetic mechanisms of adriamycin resistance and its modulation by 20(S)-ginsenoside Rh2 in MCF-7/Adr cells," British Journal of Pharmacology, vol. 165, no. 1, pp. 120-134, 2012.

[20] L. Chen, L. Liu, and W. Zhu, "Comparative pharmacokinetic studies of peimine and peiminine in rat plasma by LC-MS-MS after oral administration of Fritillaria thunbergii Miq. and Fritillaria thunbergii Miq. - Glycyrrhiza uralensis Fisch,” Die Pharmazie, vol. 66, no. 9, pp. 684-689, 2011.

[21] Z. Wang, F. Cao, Y. Chen, Z. Tang, and Z. Wang, "Simultaneous determination and pharmacokinetics of peimine and peiminine in beagle dog plasma by UPLC-MS/MS after the oral administration of Fritillariae ussuriensis maxim and Fritillariae thunbergii miq powder," Molecules, vol. 23, no. 7, p. 1573, 2018.

[22] R. Martinent, Q. Laurent, N. Sakai, and S. Matile, "Cellular uptake mediated by cyclic oligochalcogenides," CHIMIA International Journal for Chemistry, vol. 73, no. 4, pp. 304-307, 2019.

[23] J. H. Huang, L. Li, and X. Qiu, "Stimulating effects on alveolar type II epithelial A549 cells by TNF- $\alpha$ and LPS," Immunological Journal, vol. 29, no. 3, pp. 211-215, 2013. 
[24] C. L. Wu, H. Y. Wang, and J. Xu, "Magnolol inhibits tumor necrosis factor-alpha-induced ICAM-1 expression via suppression NF-kappa B and MAPK signaling pathways in human lung ithelial cells," Inflammation, vol. 37, no. 6, pp. 1957-1967, 2014.

[25] L. Y. Cao, K. Li, and W. J. Zhen, "An experimental study on the removal of high protein content in human serum by methanol precipitation," Journal of Medical Postgraduates, vol. 25, no. 11, pp. 1151-1154, 2012.

[26] J. S. Baek and C. W. Cho, "2-Hydroxypropyl- $\beta$-cyclodextrinmodified SLN of paclitaxel for overcoming p-glycoprotein function in multidrug-resistant breast cancer cells," Journal of Pharmacy and Pharmacology, vol. 65, no. 1, pp. 72-78, 2013.

[27] Guidance for Industry, Bioanalytical Method Validation, pp. 4-10, US Department of Health and Human Services Food and Drug Administration, Center for Drug Evaluation and Research (CDER), Center for Veterinary Medicine (CV), Silver Spring, MD, USA, 2001.

[28] J. C. Dong and L. X. Zeng, "Progress on application of biological sample pretreatment technology in pharmacokinetic research," Traditional Chinese Drug Research \& Clinical Pharmacology, vol. 29, no. 1, pp. 110-117, 2018.

[29] Y. Higashinoto, Y. Yamagata, T. Iwata et al., "Increased serum concentrations of tissue inhibitor of metalloproteinase-1 in COPD patients," European Respiratory Journal, vol. 25, no. 5, pp. 855-890, 2005.

[30] C. H. Ru, W. Wang, and X. Hu, "Effect of peiminine on lung function and il-8 mrna expression in peripheral blood monocytes of COPD rats," Chinese Journal of Traditional Medical Science and Technology, vol. 20, no. 3, pp. 262-263, 2013. 